\title{
Role of physical activity in mortality prediction in elderly hospice patients
}

\author{
Hye-Sang Park', Kyu-Tae Lee ${ }^{2}$, Tae-Woon Kim ${ }^{1, *}$ \\ 'Department of Physiology, College of Medicine, Kyung Hee University, Seoul, Korea \\ ¿2ohas Eun-Pyung Hyo-Hospital, Seoul, Korea
}

The prediction of life-expectancy in terminally ill patients is important both for medical and social reasons but it is widely recognized as being inaccurate. The aim of this study was to investigate the mortality predictors and indicators of life extension among elderly patients in a hospice service center. In order to determine the mortality predictors, we investigated the relationship between patients' cognitive status using the Korean-Mini Mental State Examination \& Global Deterioration Scale (K-MMSE \& GDS), the patient's physical function using the instrumental Activities of Daily Living (ADL) score, and the patient's blood component values. The subjects included 43 men and 57 women with a mean age of $82.4 \pm 8.4$ years, and a mean nursing period of $11.1 \pm 12.2$ months. These terminally ill patients were enrolled in a cross-sectional study. All data were collected from paper and electronic charts, and patient interviews. A simple correlation analysis was performed to determine the relationship between the variables and to satisfy the normal distribution $(P<0.01)$. The results revealed that the time of death negatively correlated with $A D L$ score $(r=-0.273, P=0.006)$. However, the K-MMSE \& GDS, and the values of blood component such as albumin, gamma-glutamic transpeptidase, blood urea nitrogen, and creatinine were not correlated with the time of death. Consequently, the ADL score might be an important predictor of mortality and life extension in elderly patients. Therefore, in order to improve ADL score, physical exercise and overall fitness may be potential non-pharmacologic methods useful in preventing mortality in elderly people.

Keywords: Physical activity, Activities of Daily Living, Death predictor, Elderly, Hospice patients

\section{INTRODUCTION}

The elderly population has become an important group that increasingly requires special attention with respect to health and social issues. The health of the elderly proceeds on a continuum that begins with the development of symptoms associated with biological changes, and continues to disease onset, functional loss and disability, and ultimately, terminal status and death (Rizzuto and Fratiglioni, 2014). Functional decline in the health of the elderly is associated with increased risk of mortality in terminally ill patients. Hospice care offers a highly humane and cost-effective pathway for end-of-life care (Kang et al., 2012). The purpose of hospice care is not to cure the illness, but to prolong the life expectancy of the patients. Hospice care caters to the psychological and social needs of the elderly in maintaining their quality of life, and their ability to cope with death and bereavement. This care consists of comprehensive protection service programs (Kil, 2004). However, despite the higher quality of life and better pain management that has been observed in hospice patients (Wallston et al., 1988), there is a concern that hospice care might be associated with shortened survival time (Saito et al., 2011). The prediction of life-expectancy for terminal care patients is important both for medical and social reasons, but it is widely recognized as being inaccurate. Life expectancy prediction has both emotional and practical importance for patients, family and medical staff. Patients and their families are able to request the exact information, but the ability of the medical or nursing staff to predict the endstage of a patient's life is inadequate (Addington-Hall et al., 1990). Nevertheless, it is necessary to find a predictor of mortality in order to anticipate the life expectancy of the elderly. There are
${ }^{*}$ Corresponding author: Tae-Woon Kim (D) http://orcid.org/0000-0001-8832-0874 Department of Physiology, College of Medicine, Kyung Hee University, 26 Kyungheedae-ro, Dongdaemun-gu, Seoul 02447, Korea

Tel: +82-2-961-0282, Fax: 82-2-964-2195, E-mail: twkim0806@naver.com Received: March 5, 2017 / Accepted: March 31, 2017
This is an Open Access article distributed under the terms of the Creative Commons Attribution Non-Commercial License (http://creativecommons.org/licenses/by-nc/4.0/) which permits unrestricted non-commercial use, distribution, and reproduction in any medium, provided the original work is properly cited. 
various approaches to increasing the life expectancy of the elderly once death predictors are considered. Predictors of death in the elderly are several and diverse, such as age, loneliness (Perissinotto et al., 2012), Activities of Daily Living (ADL) (Katz et al., 1963; Matzen et al., 2012; Wlodarczyk et al., 2004), recognition (Hirsch et al., 2012), quality of life (Wlodarczyk et al., 2004), physical activity (Jalayondeja et al., 2016), blood albumin (Menon et al., 2005; Sudfeld et al., 2013), and blood transfusion (Chazot et al., 2014). In this study, we investigated the predictors of mortality in elderly individuals, aiming to find ways to help prolong life expectancy. This study is based on data obtained from elderly hospice patients. The analysis of this data provides a meaningful way to explore the health and longevity of the elderly.

\section{MATERIALS AND METHODS}

\section{Participants}

One hundred elderly patients (57\% male, $43 \%$ female) who used a hospice services center in South Korea were enrolled in the study. The researchers gathered objective data on inpatient hospice patients over a period of 3 years. Since the purpose of hospice treatment is not the improvement of health or cure, no special effort was made to extend life (Table 1).

\section{Korean-Mini Mental State Examination \& Global Deterioration Scale}

We discovered a death extension index through a basic medical

Table 1. Physical characteristics of the subjects $(n=100)$

\begin{tabular}{lc}
\hline Characteristic & Value \\
\hline Sex & $1.57 \pm 0.50$ \\
Men:women & $43(43): 57(57)$ \\
Age (yr) & $82.44 \pm 8.43$ \\
Nursing period (mo) & $11.06 \pm 12.16$ \\
Cause of death & $2.22 \pm 1.62$ \\
Respiratory failure & $48(48)$ \\
Hematosepsis & $26(26)$ \\
Acute myocardial infarction & $3(3)$ \\
Pneumonia & $10(10)$ \\
Cardiogenic shock or septic shock & $5(5)$ \\
Other & $8(8)$ \\
Kind of death & $1.00 \pm 0.00$ \\
IIIness death & \\
Violent death & \\
Other &
\end{tabular}

Values are presented as mean \pm standard deviation or number $(\%)$. examination and through observation of patient records. All data were obtained from paper and electronic charts, and patient interviews. Trained research nurses entered the data with standardized definitions and coding practices. We examined the relationship between patients' cognitive status using the Korean-Mini Mental State Examination \& Global Deterioration Scale (K-MMSE \& GDS) (Wlodarczyk et al., 2004). The composition of the K-MMSE was a total of 30 points, with 10 points for orientation (time and place), 3 points for memory registration, 5 points for attention concentration and calculation, 3 points for memorial remarks, and 9 points for language and space-time. The GDS consists of 1 point for 'no cognitive impairment' to 7 points for 'late severe cognitive impairment'.

\section{Activities of Daily Living}

We examined the relationship between patients' physical functional status using the instrumental ADL score (Katz et al., 1963). Measurements of ADL performance include dressing, brushing one's teeth, bathing and hygiene related activities, eating, postural changes, movement, and using the toilet. The allotment of scores were as follows: 1 point for the fulfillment of the criteria 'complete independence' to 5 points for 'requirement of full support or inability to complete the task.'

\section{Blood component}

Blood components are total protein, albumin, aspartate transaminase (serum glutamic oxaloacetic transaminase), alanine transaminase (serum glutamic-pyruvic transaminase), gamma-glutamic transpeptidase (r-GTP), blood urea nitrogen (BUN), creatinine, sodium, potassium, chloride, glucose, hemoglobin, hamatocrit, red blood cell, white blood cell, platelet.

\section{Statistical analyses}

Three years of objective data on inpatient hospice patients were obtained. Simple correlation analysis and 2 variables all satisfy the normal distribution $(P<0.01)$. Statistical analyses were performed using IBM SPSS Statistics ver. 24.0 (IBM Co., Armonk, NY, USA).

\section{RESULTS}

\section{K- MMSE \& GDS}

MMSE total score is an average of $6.48 \pm 6.68$ points out of 30 points. GDS total score is an average of $5.68 \pm 1.46$ points out of 7 points. Cognitive ability (K-MMSE \& GDS) components are not correlated with time of death (Table 2). 


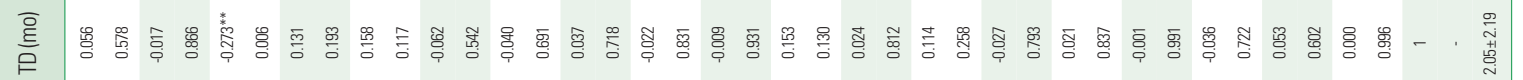

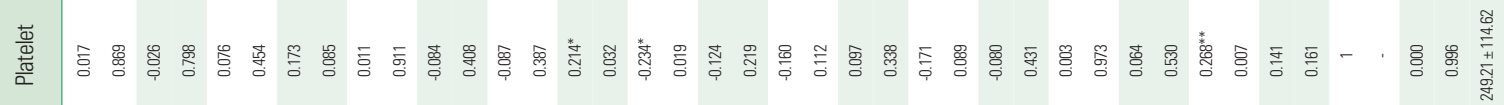

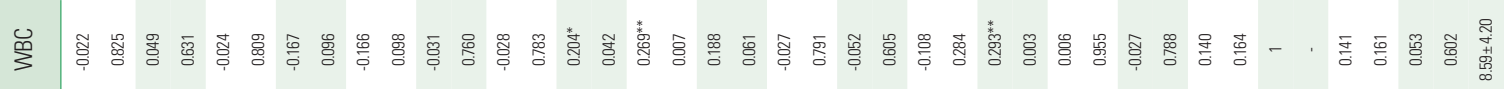

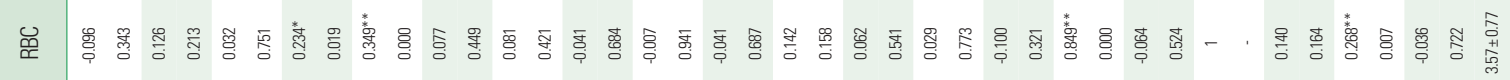

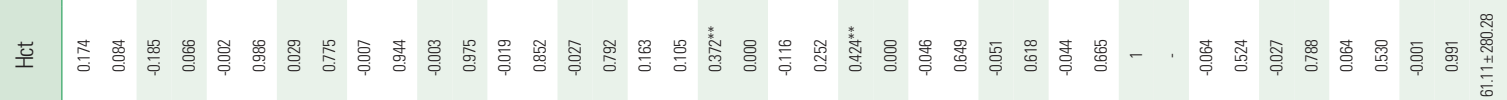

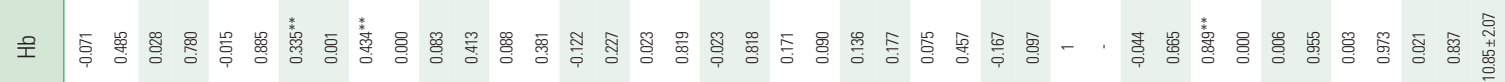

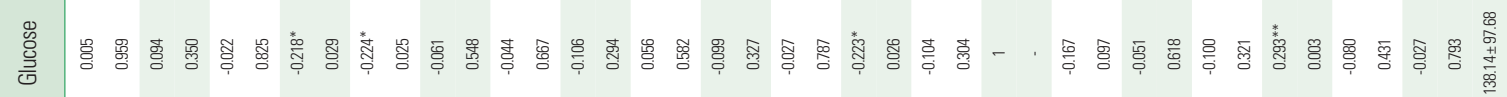

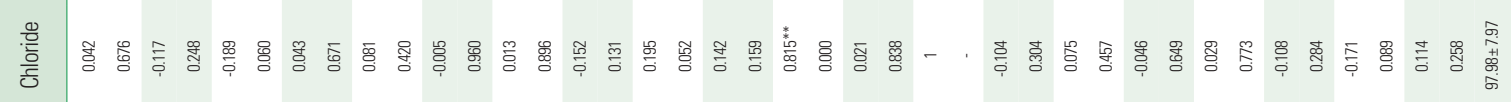

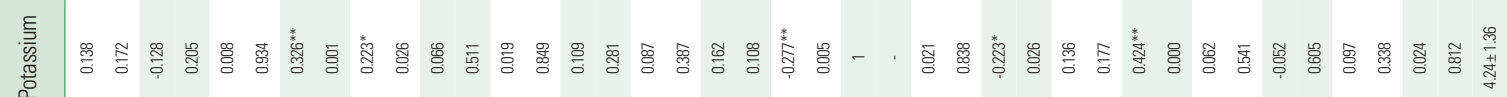

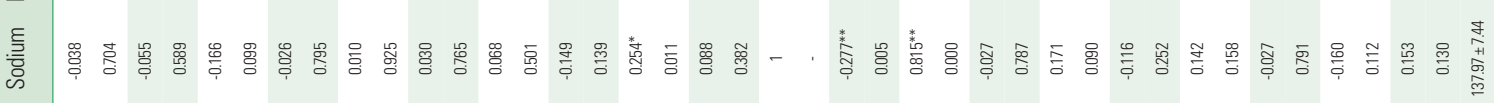

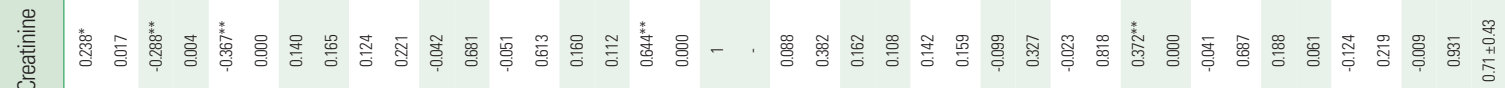

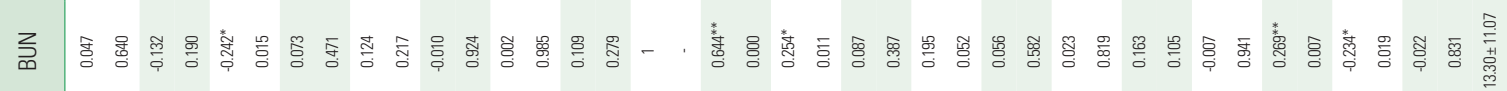

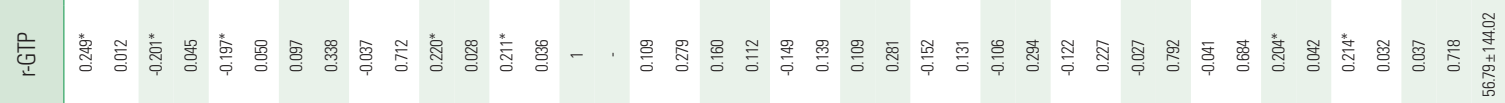

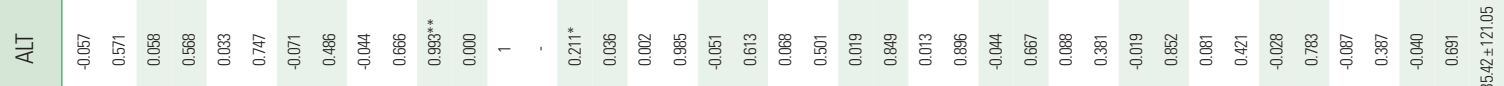

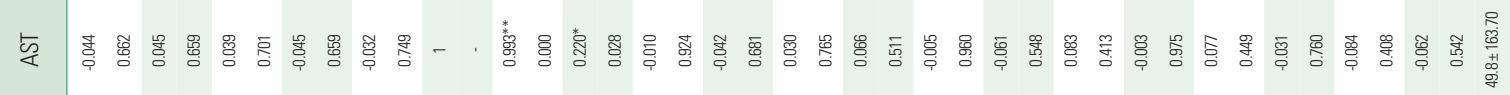

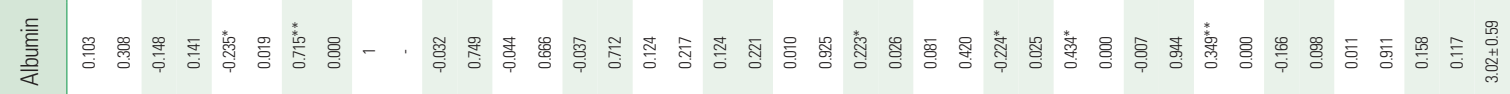

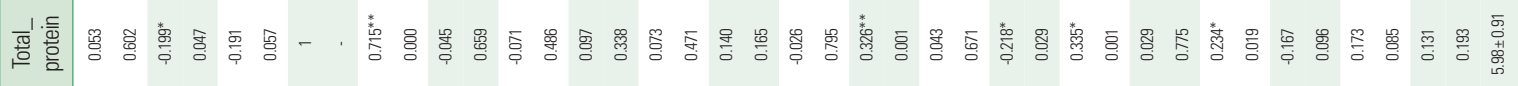

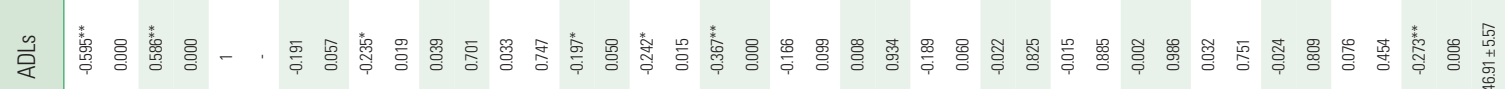

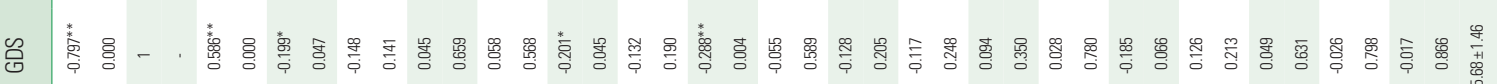

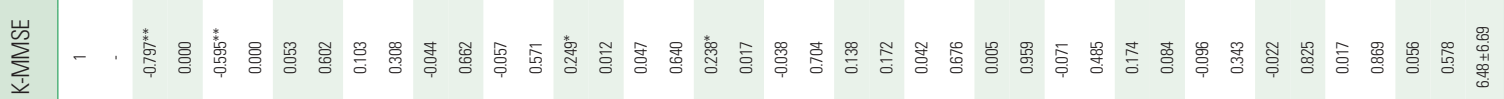

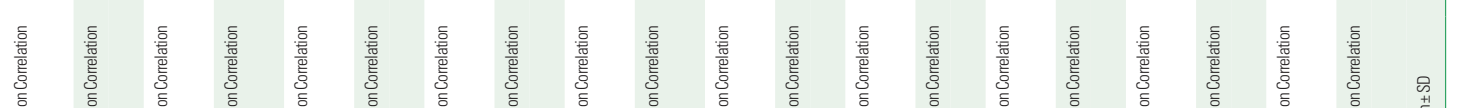

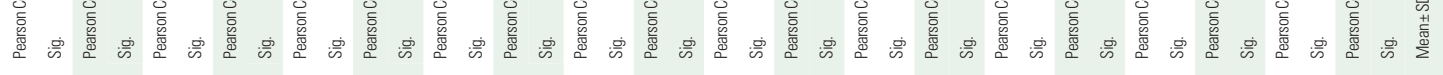

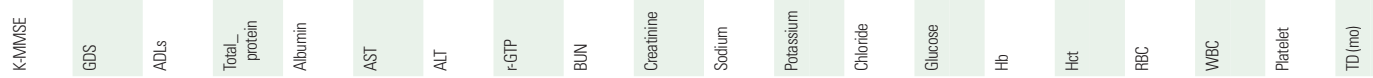




\section{Activities of Daily Living}

ADL total score is an average of $46.91 \pm 5.57$ points out of 50 points. The physical function status (ADL) is correlated with time of death in elderly hospice patient $(r=-0.273, P=0.006)$ (Table 2$)$.

\section{Blood component}

Blood component are not correlated with time of death (Table 2).

\section{DISCUSSION}

Hospice care patients receive basic medical care and observation without special rehabilitation programs to extend life. In this study, there was consistent decrease in the KMMSE \& GDS with respect to the time of death, but ADL score was even more affected to the time of death. The risk of death varied in proportion to the scores ranging from low-risk (ADL score $0-1$ ) to high-risk (ADL score 5) groups. Factors such as increasing age, dementia, stroke, and congestive heart failure were associated with higher ADL scores. Physiological measurements, such as low systolic blood pressure and high white blood cell count, were also associated with a higher ADL score (Sood et al., 2011). This suggests that the ADL score on admission captures the effect of both the acute illness and more chronic conditions, and, further, it provides a comprehensive measure of health. Therefore, ADL score is an important a predictor of mortality in the elderly. Obtaining an ADL functional score on admission was simple and easy to perform. Although the ADL score may have overestimated functionality it was strongly predictive of outcomes. This suggests that assessment of ADL score may have a potential role in decision making for the clinical management of frail elderly inpatients (Matzen et al., 2012).

ADL score was associated with blood component values in the present study. As time of death neared, ADL score decreased, and BUN, r-GTP, creatinine rose sharply. Rowe and Kahn (1997) suggested that successful aging has 3 constituent components: avoiding disease and disability, maintaining high cognitive and physical function, and engagement with life. High physical function is a crucial factor for successful aging. Measures of functional performance and physical fitness are important, simple, and objective observations of physical function in elderly. Therefore, physical activity, including exercise is needed to achieve a good ADL score. ADL have proven to be effective in improving the postprandial serum glucose in type 2 diabetic patients, but endurance exercise intensity was even more effective in improving blood sugar homeostasis than daily ADL (van Dijk et al., 2013). Long-term football exercise and strength training helped to effectively maintain the ADL score, develop muscle strength, and prevent functional decline in elderly men (Sundstrup et al., 2016). Fisher et al. (2016) suggested that early mobilization, physical therapy, walking programs, and other approaches may improve mobility in the elderly and potentially reduce hospitalization. Physical exercise and dietary measures are currently the only known ways of slowing the aging process (Le Gall and Ardaillou, 2009). Gremeaux et al. (2012) demonstrated mechanisms that underlie the positive effects of exercise on the aging process. Regular physical activity is associated with a 30\% reduction in the risk of cardiovascular mortality in subjects with cardiovascular disease (Nocon et al., 2008). This risk reduction corresponds to $1-2$ years of additional life attributable to adequate exercise when compared to individuals who engage in little or no physical activity (Franco et al., 2005). However, the study of exercise intensity for the elderly can be divided into various segments. Xue et al. (2012) suggested that physical activity does not have to be vigorous to be beneficial and that the gain may be the greatest among women who reported the lowest levels of activity. In contrast, Samitz et al. (2011) demonstrated that larger training volume (exercise duration $\times$ intensity) is associated with greater reduction in mortality. For a given training volume, engaging in physical activities of a higher intensity provides additional benefit.

According to the statement of the hospital staff, this improvement was related to a correction in dehydration, improved chewing ability, and a drug regimen, among other interventions. As the patient neared death, all functional abilities declined at various rates. Until death, cognitive ability (K-MMSE \& GDS) did not decrease as much, but physical functional status and ADL score decreased, and blood component values worsened. In conclusion, ADL scores might be seen as indicators for the delaying of death. Thus, when providing care to those confronted with terminal illness it is important to increase the ADL score. The method to achieve this is to increase physical activity. Consequently, in order to improve ADL score, physical exercise and improved fitness are potential nonpharmacologic means to prevent mortality in the elderly. Using objective data, the present study confirmed some of the clinical reports of medical and nursing staff working in the hospice field; however, further study is needed to construct a model that enables prediction of life-expectancy on the basis of the objective parameters.

\section{CONFLICT OF INTEREST}

No potential conflict of interest relevant to this article was reported. 


\section{REFERENCES}

Addington-Hall JM, MacDonald LD, Anderson HR. Can the Spitzer Quality of Life Index help to reduce prognostic uncertainty in terminal care? Br J Cancer 1990;62:695-699.

Chazot C, Deleaval P, Bernollin AL, Vo-Van C, Lorriaux C, Hurot JM, Mayor B, Jean G. Target weight gain during the first year of hemodialysis therapy is associated with patient survival. Nephron Clin Pract 2014;126:128-134.

Fisher SR, Graham JE, Ottenbacher KJ, Deer R, Ostir GV. Inpatient walking activity to predict readmission in older adults. Arch Phys Med Rehabil 2016;97(9 Suppl):S226-231.

Franco OH, de Laet C, Peeters A, Jonker J, Mackenbach J, Nusselder W. Effects of physical activity on life expectancy with cardiovascular disease. Arch Intern Med 2005;165:2355-2360.

Gremeaux V, Gayda M, Lepers R, Sosner P, Juneau M, Nigam A. Exercise and longevity. Maturitas 2012;73:312-317.

Hirsch CH, Buzková P, Robbins JA, Patel KV, Newman AB. Predicting late-life disability and death by the rate of decline in physical performance measures. Age Ageing 2012;41:155-161.

Jalayondeja C, Jalayondeja W, Suttiwong J, Sullivan PE, Nilanthi DL. PhysiCAL Activity, self-esteem, and quality of life among people with physical disability. Southeast Asian J Trop Med Public Health 2016; 47:546-558.

Kang SC, Lin MH, Hwang IH, Lin MH, Chang HT, Hwang SJ. Impact of hospice care on end-of-life hospitalization of elderly patients with lung cancer in Taiwan. J Chin Med Assoc 2012;75:221-226.

Katz S, Ford AB, Moskowitz RW, Jackson BA, Jaffe MW. Studies of illness in the aged. The index of adl: a standardized measure of biological and psychosocial function. JAMA 1963;185:914-919.

Kil GS. A study on the quality of life related to care and hospice services for the elderly with chronic illness. J welfare aged 2004;24;239-275.

Le Gall JY, Ardaillou R. The biology of aging. Bull Acad Natl Med 2009; 193:365-402.

Matzen LE, Jepsen DB, Ryg J, Masud T. Functional level at admission is a predictor of survival in older patients admitted to an acute geriatric unit. BMC Geriatr 2012;12:32.

Menon V, Greene T, Wang X, Pereira AA, Marcovina SM, Beck GJ, Kusek JW, Collins AJ, Levey AS, Sarnak MJ. C-reactive protein and albumin as predictors of all-cause and cardiovascular mortality in chronic kidney disease. Kidney Int 2005;68:766-772.

Nocon M, Hiemann T, Müller-Riemenschneider F, Thalau F, Roll S, Willich SN. Association of physical activity with all-cause and cardiovascular mortality: a systematic review and meta-analysis. Eur J Cardio- vasc Prev Rehabil 2008;15:239-246.

Perissinotto CM, Stijacic Cenzer I, Covinsky KE. Loneliness in older persons: a predictor of functional decline and death. Arch Intern Med 2012;172:1078-1083.

Rizzuto D, Fratiglioni L. Lifestyle factors related to mortality and survival: a mini-review. Gerontology 2014;60:327-335.

Rowe JW, Kahn RL. Successful aging. Gerontologist 1997;37:433-440.

Saito AM, Landrum MB, Neville BA, Ayanian JZ, Weeks JC, Earle CC. Hospice care and survival among elderly patients with lung cancer. J Palliat Med 2011;14:929-939.

Samitz G, Egger M, Zwahlen M. Domains of physical activity and allcause mortality: systematic review and dose-response meta-analysis of cohort studies. Int J Epidemiol 2011;40:1382-1400.

Sood MM, Rigatto C, Bueti J, Jassal V, Miller L, Verrelli M, Bohm C, Mojica J, Roberts D, Komenda P. The role of functional status in discharge to assisted care facilities and in-hospital death among dialysis patients. Am J Kidney Dis 2011;58:804-812.

Sudfeld CR, Isanaka S, Aboud S, Mugusi FM, Wang M, Chalamilla GE, Fawzi WW. Association of serum albumin concentration with mortality, morbidity, CD4 T-cell reconstitution among tanzanians initiating antiretroviral therapy. J Infect Dis 2013;207:1370-1378.

Sundstrup E, Jakobsen MD, Andersen LL, Andersen TR, Randers MB, Helge JW, Suetta C, Schmidt JF, Bangsbo J, Krustrup P, Aagaard P. Positive effects of 1-year football and strength training on mechanical muscle function and functional capacity in elderly men. Eur J Appl Physiol 2016;116:1127-1138.

van Dijk JW, Venema M, van Mechelen W, Stehouwer CD, Hartgens F, van Loon LJ. Effect of moderate-intensity exercise versus activities of daily living on 24-hour blood glucose homeostasis in male patients with type 2 diabetes. Diabetes Care 2013;36:3448-3453.

Wallston KA, Burger C, Smith RA, Baugher RJ. Comparing the quality of death for hospice and non-hospice cancer patients. Med Care 1988;26: 177-182.

Wlodarczyk JH, Brodaty H, Hawthorne G. The relationship between quality of life, Mini-Mental State Examination, and the Instrumental Activities of Daily Living in patients with Alzheimer's disease. Arch Gerontol Geriatr 2004;39:25-33.

Xue QL, Bandeen-Roche K, Mielenz TJ, Seplaki CL, Szanton SL, Thorpe RJ, Kalyani RR, Chaves PH, Dam TT, Ornstein K, RoyChoudhury A, Varadhan R, Yao W, Fried LP. Patterns of 12-year change in physical activity levels in community-dwelling older women: can modest levels of physical activity help older women live longer? Am J Epidemiol 2012;176:534-543. 